\title{
An investigation of tomotherapy beam delivery
}

\author{
James N. Yang ${ }^{\text {a) }}$ \\ Department of Medical Physics, University of Wisconsin Medical School, Madison, Wisconsin 53706
}

T. Rock Mackie

Departments of Medical Physics and Human Oncology, University of Wisconsin Medical School, Madison, Wisconsin 53706

Paul Reckwerdt

Department of Medical Physics, University of Wisconsin Medical School, Madison, Wisconsin 53706

Joseph O. Deasy ${ }^{\mathrm{b})}$ and Bruce R. Thomadsen

Departments of Medical Physics and Human Oncology, University of Wisconsin Medical School, Madison, Wisconsin 53706

(Received 13 March 1996; accepted for publication 11 December 1996)

\begin{abstract}
Experimental simulations for tomotherapy beam delivery were performed using a computercontrolled phantom positioner, a cylindrical phantom, and a $6 \mathrm{MV}$ x-ray slit beam. Both continuous helical beam and sequential segmented tomotherapy (SST) beam deliveries were evaluated. Beam junctioning problem due to couch indexing error or field width errors presented severe dose uniformity perturbations for SST, while the problem was minimized for helical beam delivery. Longitudinal breathing motions were experimentally simulated for helical and SST beam delivery. While motions reduced the dose uniformity perturbations for SST, small artifacts in dose uniformity can be introduced for helical beam delivery. With typical breath frequency and magnitude, for a slit beam of $2.0 \mathrm{~cm}$ width at $4 \mathrm{rpm}$, the dose uniformity perturbation was not significant. A running start/stop technique was implemented with helical beam delivery to sharpen the $20 \%-80 \%$ longitudinal dose fall-off from 1.5 to $0.5 \mathrm{~cm}$. The latter was comparable to the corresponding dose penumbra of a conventional $6 \mathrm{MV} 10 \times 10 \mathrm{~cm}^{2}$ field. All together, helical beam delivery showed advantages over SST for tomotherapy beam delivery under similar delivery conditions. (C) 1997 American Association of Physicists in Medicine. [S0094-2405(97)00803-1]
\end{abstract}

Key words: tomotherapy, conformal radiotherapy, helical beam, motion artifacts

\section{INTRODUCTION}

Much effort in radiotherapy has been devoted toward inverse planning, employing modulated intensity beams to achieve conformal dose distributions. ${ }^{1-14}$ Mackie et al. proposed a "tomotherapy" system. ${ }^{15}$ It included inverse treatment planning, dose delivery, and verification subsystems for dynamic conformal radiotherapy. It would use a slit beam emerging from a temporally modulated multileaf collimator (TMMLC) system. The dose to the target would be delivered in a helical fashion using continuous gantry rotations and simultaneous patient longitudinal translations similar to motions in helical (or spiral) CT. As a result of the slit beam, only a part of the target would be irradiated at a given moment during the treatment. The dose to the target would be delivered with the slit beam wrapping around the target in contrast to the conventional approach, in which the whole target is enclosed in the projection of a large static beam. A TMMLC system, the Peacock system (NOMOS Corporation, Sewickley, PA), is commercially available as an add-on component to conventional medical linacs. ${ }^{16}$ The Peacock system was designed to

\footnotetext{
${ }^{a}$ Presently at the Southern Wisconsin Radiotherapy Center, Madison, Wisconsin 53713

${ }^{b}$ Presently at the Brown Cancer Center, University of Louisville, Louisville, Kentucky 40202.
}

be used for conformal treatment with sequential gantry rotation and couch longitudinal translation in the fashion of a traditional CT scanner. Two sets of collimator leaves allow two adjacent slices of the target to be treated simultaneously. The couch is then translated a distance of two segment widths (two slit widths) longitudinally to treat the next segment of the target. This form of radiation treatment is referred to as the sequential segmented tomotherapy (SST) in this paper.

Local failure can be caused not only by an insufficiently prescribed radiation dose delivered to the tumor, but also the inadequate geometric coverage of the tumor region by radiation. ${ }^{17}$ Patient or organ motion are factors that may cause inadequate dose coverage of a target. ${ }^{18,19}$ These organ motions may pose an obstacle for intensity modulation using tomotherapy or other proposed methods of nonuniform beam delivery. ${ }^{20-23}$ When a target is irradiated by a narrow slit beam, motions cause the targeted segment to cross the beam and may cause nonuniform dose distributions and/or inadequate dose coverage within the target, resulting in local failure. In this regard, the superior-inferior motion is most important for tomotherapy beam delivery because the patient would be translated longitudinally. The lateral motion issues are not much different from those treated with a large static field. 


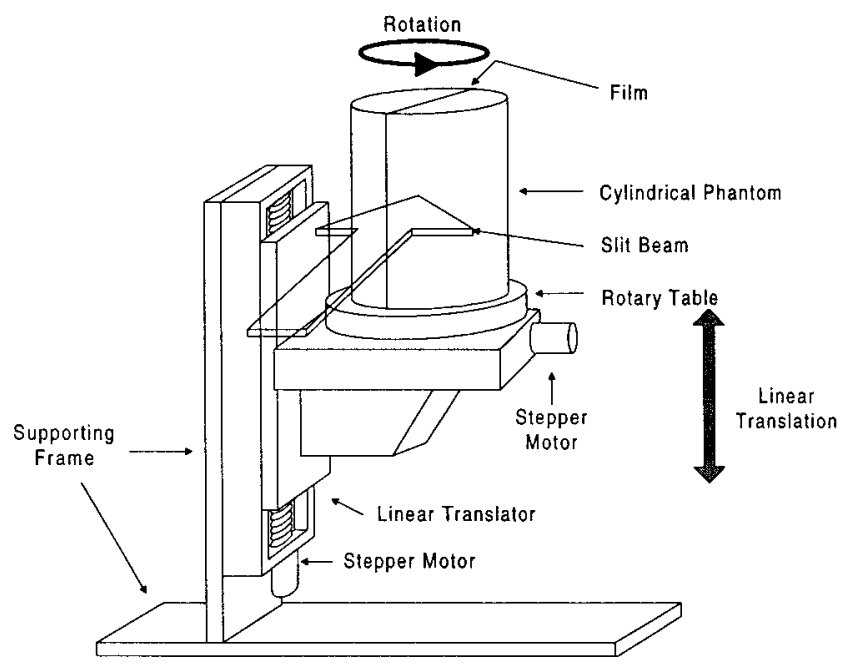

FIG. 1. A schematic drawing of the phantom positioning device used to simulate continuous helical beam delivery and sequential rotational beam delivery.

If tomotherapy were successfully implemented, it would provide an integrated means of three-dimensional (3D) conformal planning, dose delivery, and dose verification. Experimental evaluations of the beam delivery system are necessary to demonstrate its feasibility.

In this work we focused on the dosimetric characteristics for helical tomotherapy. We also studied SST delivery, with beam size defined by the linac jaws, for comparison. Procedures and devices have been developed to simulate tomotherapy helical beam delivery and SST using a uniform x-ray beam from a conventional medical linear accelerator.

\section{METHODS AND MATERIALS}

\section{A. The computer-controlled phantom positioning device}

A computer-controlled phantom positioning device was designed and constructed to aid in delivering continuous helical and SST radiation beams to a phantom (see Figs. 1 and 2) with a static horizontal slit beam. The linear motion was in the vertical direction. The phantom was rotated around a vertical axis. The system consisted of a Daedal (Daedal Division, Parker Hannifin Corporation, Harrison City, PA) 25 $\mathrm{cm}$ diameter rotary table, a Daedal linear stage with a $28 \mathrm{~cm}$ traveling length. All the movements and positions on each motion axis were driven by a computer-controlled microstep motor. The accuracy of the linear stage and the turntable were stated by the manufacturer to be $5 \mu \mathrm{m}$ and $3 \mathrm{mrad}$, respectively. ${ }^{24}$ This device may be loaded with up to $45 \mathrm{~kg}$ when the center of gravity is on the rotational axis.

\section{B. Cylindrical phantom}

A cylindrical water phantom (Fig. 2) was constructed specifically for film dosimetry. It consisted of two closed halfcylindrical polymethyl methacrylate (acrylic) shells. Each was fully filled with water. The Lucite shell was $0.6 \mathrm{~cm}$ thick

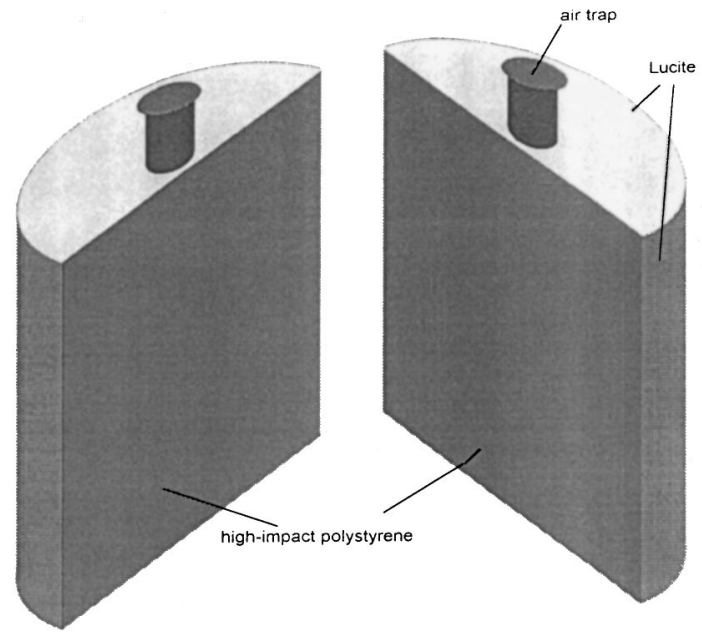

FIG. 2. A schematic drawing of the cylindrical water phantom.

and $23 \mathrm{~cm}$ long with a $18 \mathrm{~cm}$ diameter. Each flat surface piece that passed through the cylindrical central axis was made of $0.3 \mathrm{~cm}$ thick high-impact polystyrene (with an electron density of 1.02 times that of water) in order to minimize the changes in photon and electron transport between the water and the phantom material near the measurement site. An air trap on the top of each half-cylinder allowed complete filling of water by eliminating air bubbles. A ready-packed Kodak XV film was placed between the two half-cylinders. The two half-cylinders were then held with a ring clamp at each end, resulting in a full cylindrical phantom. Although there is depth dependence in film response for a single $\mathrm{x}$-ray beam due to the differential energy response, Holmes has shown that there was little depth dependency in response to opposing beams, ${ }^{25}$ which comprises the rotational beams such as SST and helical beams, as described in this work. This could be explained if the energy spectra from opposed beams compensate each other in a way to produce similar film responses, regardless of depth. The exposed films were scanned on a Scanditronix RFA-300 data acquisition system with a $1.0 \mathrm{~mm}$ aperture (Scanditronix, Newburyport, MA). A signal-to-dose film response curve was measured for each experiment to convert densitometric readings to dose. Film dosimetry was used to obtain relative dose distributions. The dose values were normalized to the central point value of the same film when it was a uniform distribution. When dose distributions from different films are compared, energy conservation law was used wherever applicable. Otherwise, the relative dose values of a dose profile were normalized to a point, within the profile, least deviated from a uniform distribution.

\section{Helical tomotherapy simulation}

A continuous helical beam was simulated using our computer-controlled phantom positioning device (Fig. 1) and cylindrical phantom (Fig. 2). The motion-control computer was located outside the treatment room. A helical beam was 
delivered by simultaneously rotating and translating the phantom along the phantom central axis while a horizontal slit radiation beam was turned on. The gantry angle of a Varian (Varian Associates, Palo Alto, CA) Clinac 2100C was kept at either $90^{\circ}$ or $270^{\circ}$. The collimator was set such that the narrow dimension of the slit field was along the vertical direction. The cylindrical phantom axis was positioned vertically on the rotary table of the computercontrolled phantom positioner. The phantom cylindrical axis coincided with the rotational axis of the rotary table. While the phantom was rotating and vertically advancing, the static slit beam continuously irradiated the phantom until the desirable total treatment length was reached. To avoid confusion, the term "ffield width" is referred to the field dimension along the cylindrical axis of the phantom, whereas "field length" is referred to the field dimension perpendicular to the field width at the same distance from the x-ray source. The field size is expressed as length $\times$ width. After each rotation, the phantom translated a distance equal to one field width. The total length of the treated region would be

$$
L=(n-1) \cdot l,
$$

where $L$ is the total longitudinal length of the region fully irradiated, $n$ is the number of rotations delivered, and $l$ is the slit beam width.

A slit beam of $8.0 \mathrm{~cm} \times 2.0 \mathrm{~cm}$ was used in most experiments. The phantom was rotated at $4 \mathrm{rpm}$. The relation between the rotational motion and the linear motion was such that the phantom was translated one field width longitudinally upon a completion of one rotation (i.e., using helical CT terms; a pitch of one).

\section{Sequential segmented tomotherapy (SST) simulation}

As a possible alternative to helical beam delivery, the SST was simulated with the same experimental apparatus in a similar manner, except that there was no simultaneous rotation and translation for SST. The slit beam was defined by the jaws in the linac used in this work. The phantom was longitudinally advanced by one field width to the next segment only after the completion of a rotation and before the start of the next rotation. The beam was off during the longitudinal translations between the segments.

\section{E. Theoretical computation}

In addition to experimental measurements, theoretical calculations were performed for the longitudinal dose profiles along the central axis of the cylindrical phantom for both the helical and SST beams (without target motion). The calculation was based on a one-dimensional convolution. In a homogeneous medium, the dose absorbed, $D$, at point $x$ from fields translated in the medium can be calculated by the following convolution equation:

$$
D(x)=\int S\left(x^{\prime}, a\right) K\left(x-x^{\prime}, b\right) d x^{\prime},
$$
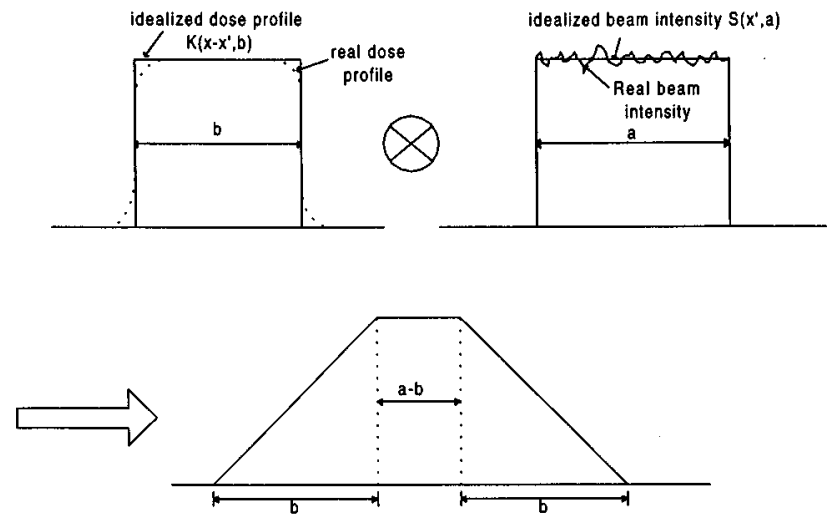

FIG. 3. A diagram for the convolution of an ideal slit beam dose profile (upper left panel) and an ideal beam intensity distribution (upper right panel). The result dose distribution is shown in the lower panel, where $a>b$.

where $S\left(x^{\prime}, a\right)$ is a source function that represents a distribution of such beams along the longitudinal axis, with the central axis at $x^{\prime}$ and a distribution range $a . K\left(x-x^{\prime}, b\right)$ is a one-dimensional convolution kernel representing the dose profile or distribution along the longitudinal axis from a single beam of width $b$. For a fixed collimation, the kernel $K$ was a measured central longitudinal dose distribution from a single static beam used to produce the helical and/or sequential beams in the cylindrical phantom. The source function $S$ was the distribution of the beams along the central axis of the cylindrical phantom. Twenty beams per rotation distributed evenly in angle were used for our calculation. Since each beam would have the same irradiation time, they were equally weighted. As shown in Fig. 3, for an ideal square kernel and source functions, the resulting dose distribution has a flat top with linear fall-off sides.

\section{F. Experimental motion simulation}

Patient longitudinal thoracic motions due to breathing were simulated with the computer-controlled phantom positioning device. Several different combinations of motion magnitude and frequency were used in our simulations. The motions were simulated experimentally along the phantom longitudinal axis with relative velocity between the slit beam and the target for both sequential rotational beams and helical rotational beams.

To determine reasonable values of breathing motion parameters, we studied video records of fluoroscopic images for patients with gold seeds implanted in the thoracic region. The superior-inferior motion magnitude (peak-to-valley) was mostly under or near $1.0 \mathrm{~cm}$, occasionally exceeding 1.0 $\mathrm{cm}$. Deviations in displacement were varied from this experimental nominal value. The typical breath frequencies we observed were 10-15 oscillations/min, but frequencies between 5 and 20 oscillations/min were investigated.

\section{G. Simulation of the running start/stop procedure}

To sharpen the dose gradient at the beginning and the end of the target, a technique called "running start/stop," similar 


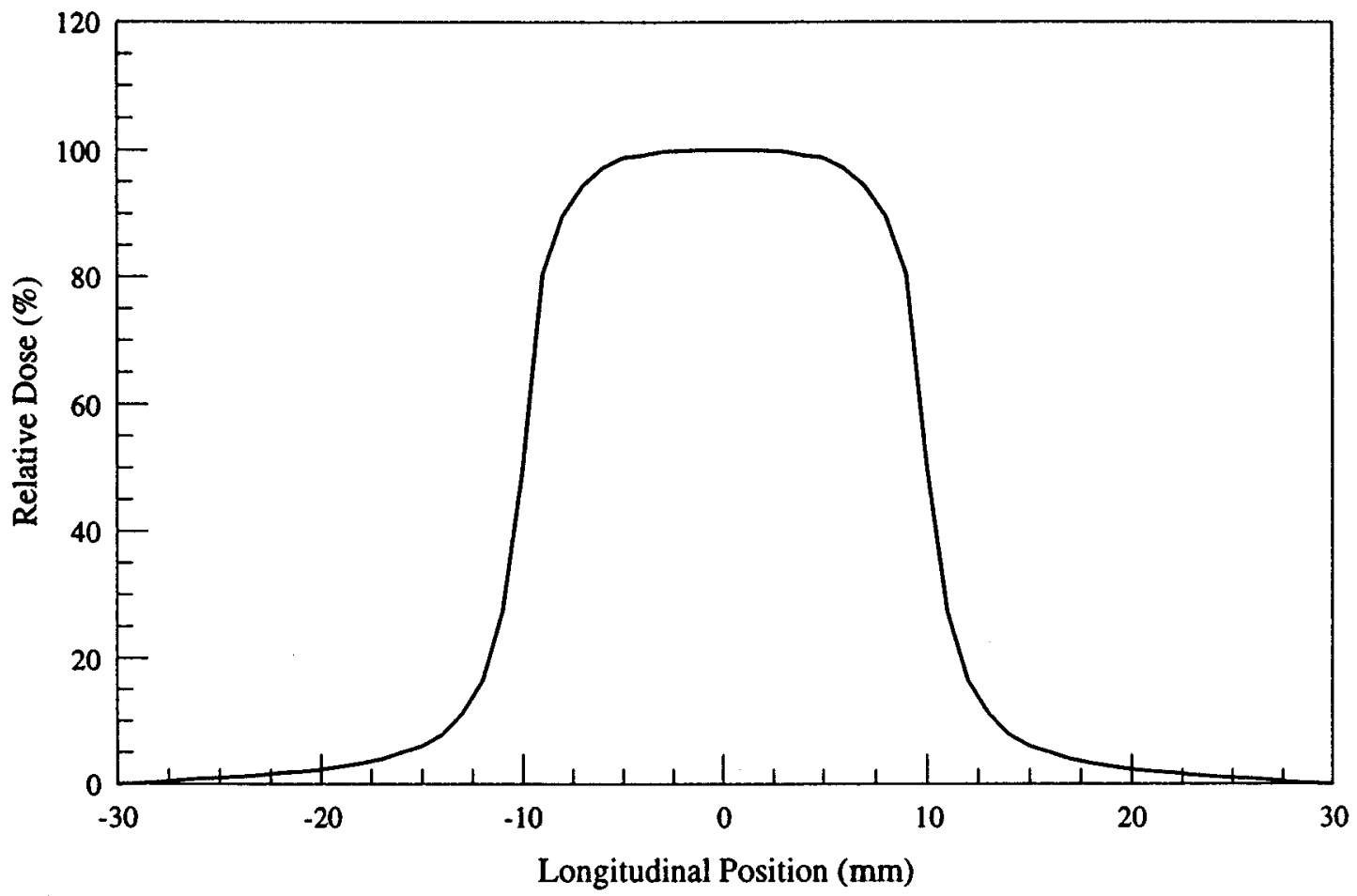

FIG. 4. The measured longitudinal dose profile along the phantom axis of a single $8.0 \mathrm{~cm} \times 2.0 \mathrm{~cm}$ beam. It served as a convolution kernel in dose profile calculations (see Fig. 5).

to a technique used in a tracking Cc-60 machine, was proposed. ${ }^{26-28}$ In the running start, the inferior collimator is opened at a predetermined rate, from a fully closed status, while the phantom longitudinal position remains unchanged until the jaw is fully opened. During the running stop, both the phantom longitudinal position and the inferior jaw are kept unchanged, and the superior jaw is closed gradually toward the inferior jaw at a predetermined rate. This technique was introduced to sharpen the dose fall-off at the longitudinal edges of a target for the helical beam delivery. However, a continuous running start/stop implementation requires dynamic control of jaw positions, which was not available. A series of static slit beams, as described below, was implemented for dosimetric evaluation. The independent jaw feature on the Varian Clinac 2100C was utilized.

At the beginning of the helical beam running start, the beam slit width was set at $2.0 \mathrm{~mm}$ for the first beam. A fixed amount of radiation was given at this position. After the completion of irradiation at this position, the inferior jaw was extended $2 \mathrm{~mm}$, as measured at the isocenter, while the phantom rotated to the next position. This was repeated until the jaw was fully opened to its desirable width. The initial jaw position was set in such a way that when the jaws were fully opened, the jaw pair was symmetric about the central ray of the beam. During the last rotation of the beam, the running stop was implemented. The superior jaw was reduced $2 \mathrm{~mm}$ after each rotational movement until the field length reached $2 \mathrm{~mm}$. Ten even angularly distributed positions were chosen and eight monitor units were given for each of these angular positions. No differential output factors were applied for the different field sizes used. There was no linear translation of the phantom during the running start/end processes. Between the running start/end, the phantom was under continuous helical motion while the beam was on. A $8.0 \mathrm{~cm} \times 2.0 \mathrm{~cm}$ field was used during this phase. The continuous helical motion was such that the distance translated equals the slit width after completion of each rotation (pitch of 1).

\section{RESULTS}

Figure 4 is a measured longitudinal dose profile, along the phantom central axis, of a static beam. The field size was 8.0 $\mathrm{cm} \times 2.0 \mathrm{~cm}$ at the isocenter. The source-to-phantom axis distance was $100 \mathrm{~cm}$ and $20 \mathrm{MU}$ was irradiated for the single field. Figure 5 shows a measured central longitudinal dose profile for one rotation of a helical beam, together with a corresponding profile calculated using the one-dimensional convolution. The dose profile of the single beam in Fig. 4 was used as the kernel for the calculation. The source distribution consisted of 20 beams per rotation, evenly distributed angularly and longitudinally. The central ray of each beam was $1.0 \mathrm{~mm}$ apart. Also shown is a triangle function that is the result described in Fig. 3 if the dose profile for a single beam was rectangular and the beam distribution was constant over the targeted region.

The curves shown in Fig. 6 are measured and calculated central longitudinal dose profiles for helical beam simulations for multiple rotations. The calculated profile was obtained using Eq. (2). The beam size is again $8.0 \mathrm{~cm} \times 2.0 \mathrm{~cm}$. 


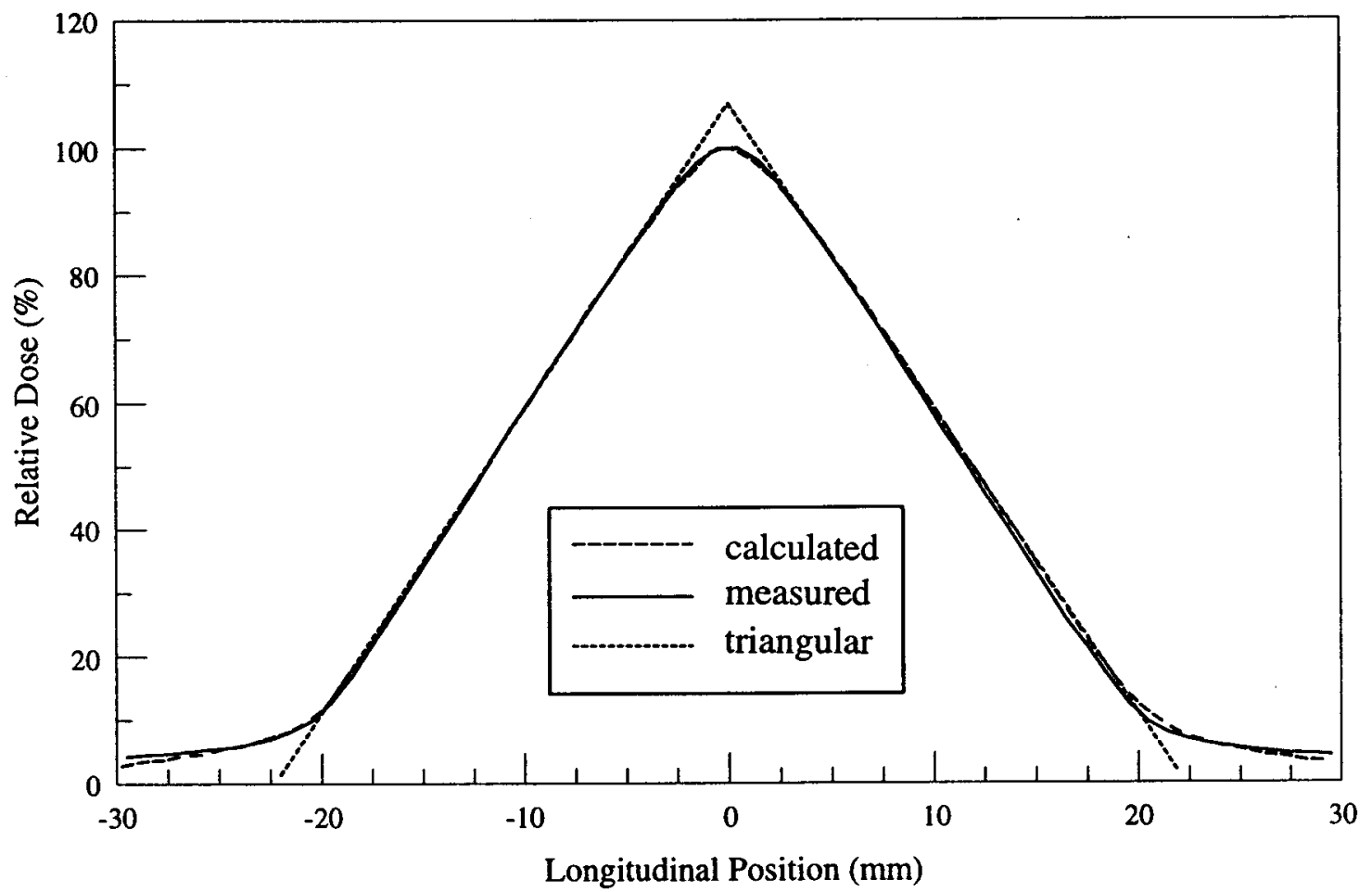

FIG. 5. The longitudinal dose profiles along the phantom axis for one helical rotation. The field size was $8.0 \mathrm{~cm} \times 2.0 \mathrm{~cm}$. The calculated and measured profiles are nearly identical. Also shown for comparison is a triangular function.

The phantom was rotated at $4 \mathrm{rev} / \mathrm{min}$ with simultaneous translation at a speed of $2.0 \mathrm{~cm} / \mathrm{rev}$. Forty MU were delivered for each rotation of the helical beam. The off-axis relative dose profiles were nearly identical to those shown here.
Figure 7 shows the calculated longitudinal dose profiles for helical beams with slit widths of $1.9,2.0$, and $2.1 \mathrm{~cm}$. They had the same field length of $8 \mathrm{~cm}$. The dose values in all three profiles were normalized to the central point value of

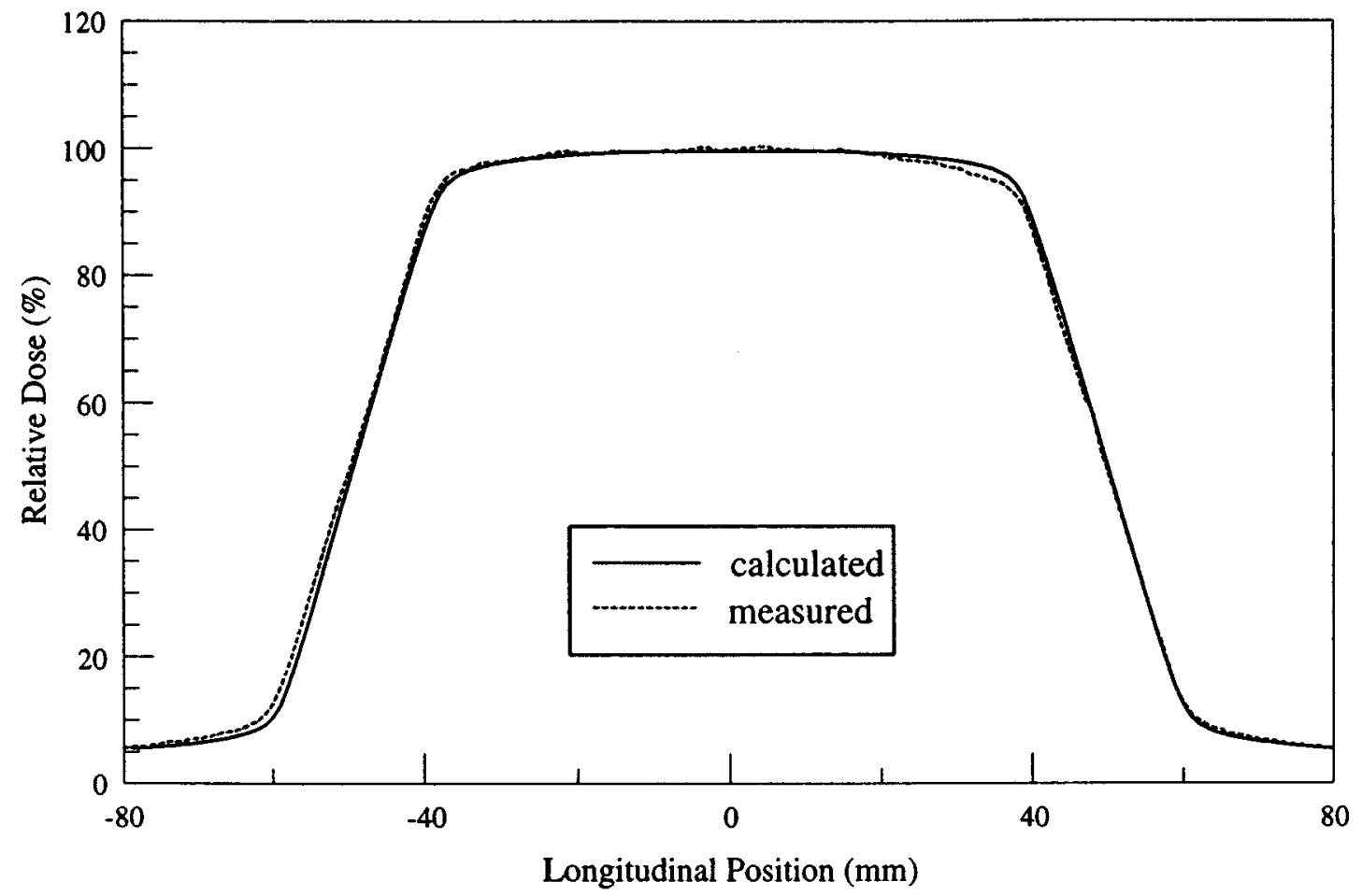

FIG. 6. Longitudinal dose profile along the phantom axis for continuous helical delivery. Field size: $8.0 \mathrm{~cm} \times 2.0 \mathrm{~cm}$. The longitudinal translation was 2.0 $\mathrm{cm} /$ rotation for a pitch of 1.0 . The running start/stop of the jaws defining the slit width was not performed. 


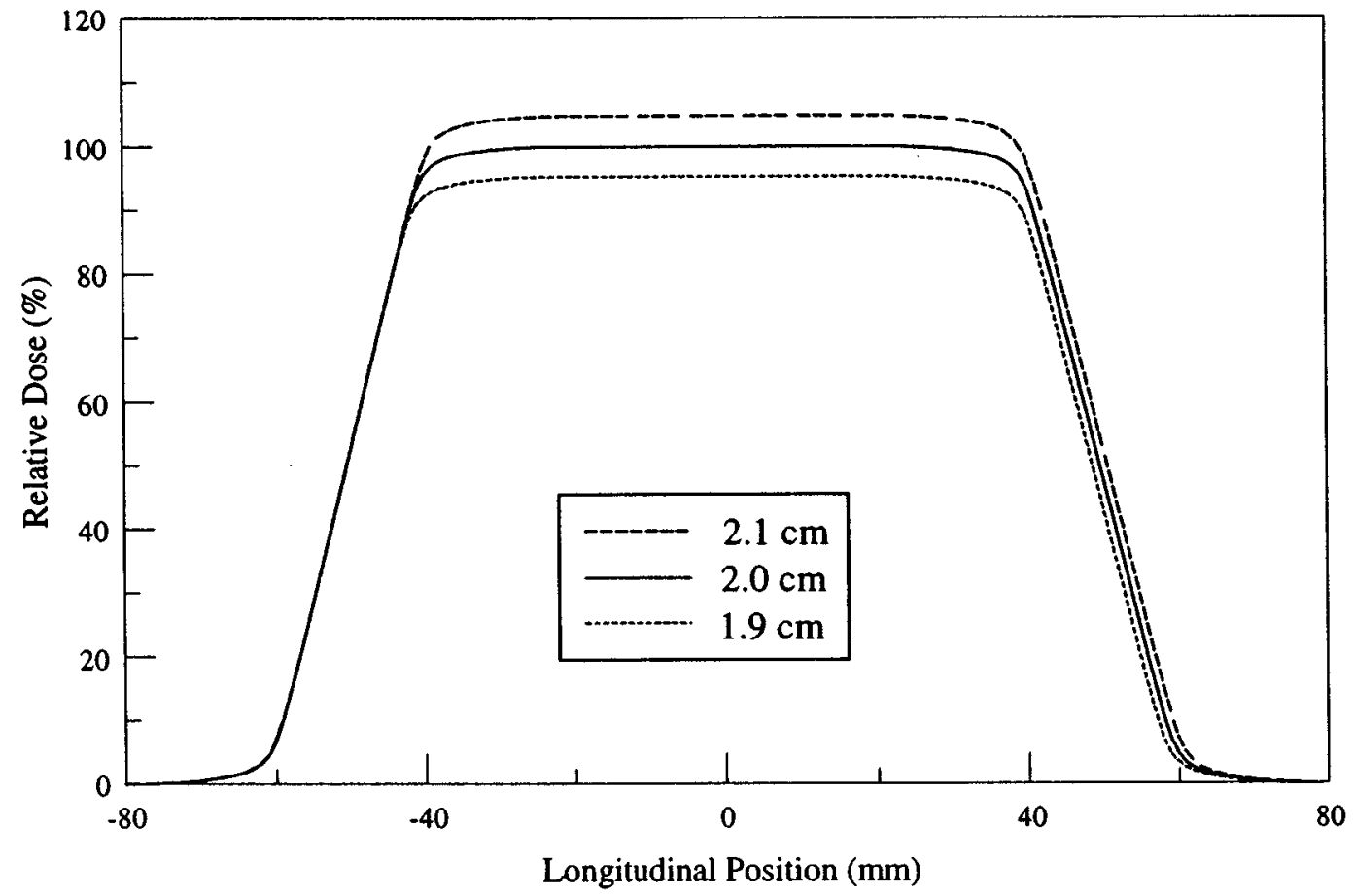

Fig. 7. The calculated helical beam dose profiles for slightly different slit widths: $2.1,2.0$, and $1.9 \mathrm{~cm}$. The field length is $8 \mathrm{~cm}$. The relative dose values are normalized to the dose at the center of the profile for $2.0 \mathrm{~cm}$ slit width.

the profile for $2.0 \mathrm{~cm}$ slit width. The relative longitudinal dose profile (when normalized to its own profile center) was not sensitive to the field width. A $1.0 \mathrm{~mm}$ increase or decrease in field width from $2.0 \mathrm{~cm}$ resulted in the same rela- tive profile. However, the absolute dose level at the central region differed by about $5 \%$ when the same amount of monitor units were given.

Figure 8 shows two measured transverse dose profiles for

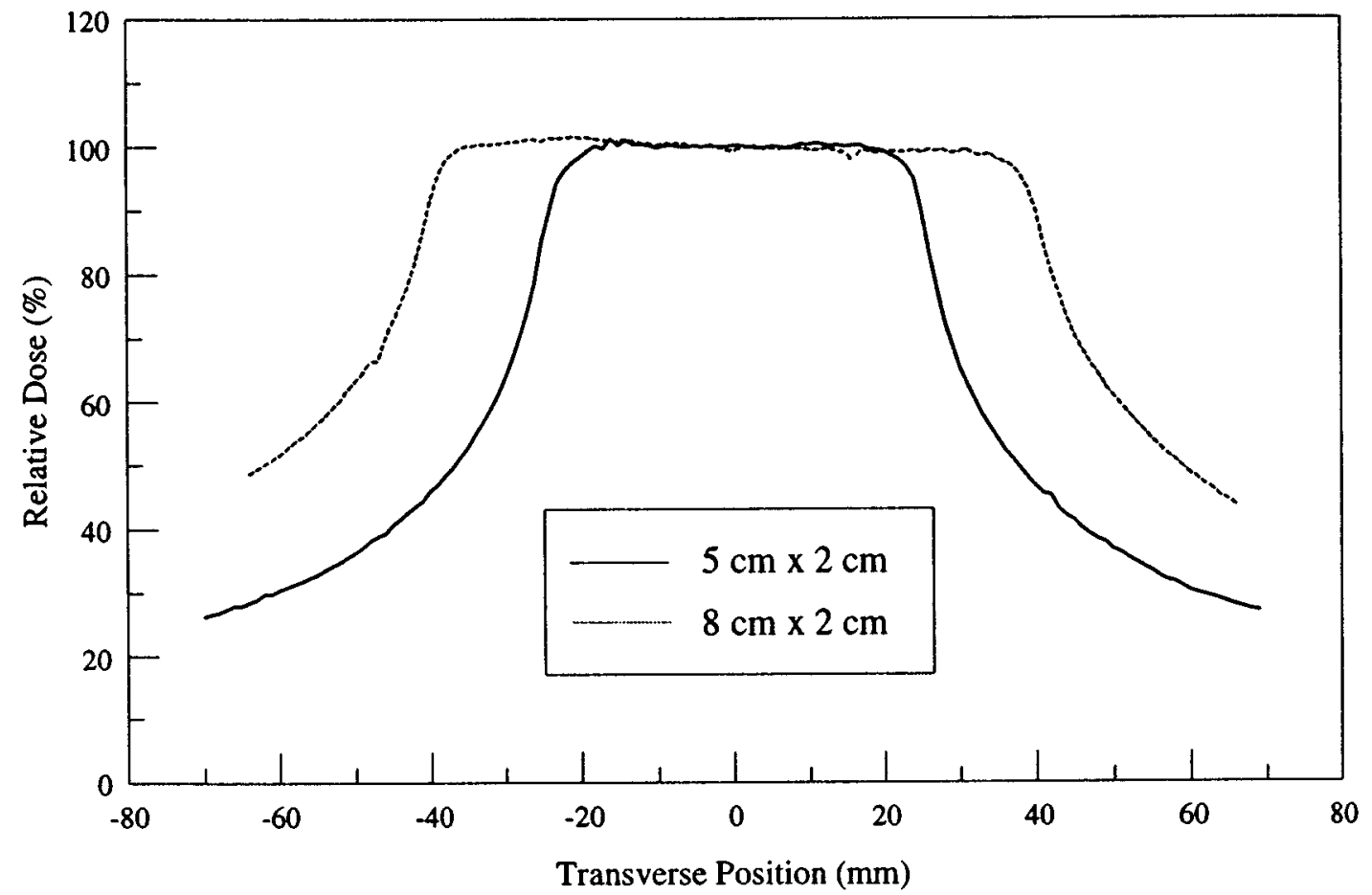

Fig. 8. Transverse (perpendicular to the phantom axis with the origin passing through the axis) dose profiles for continuous helical beam delivery with different field sizes. 


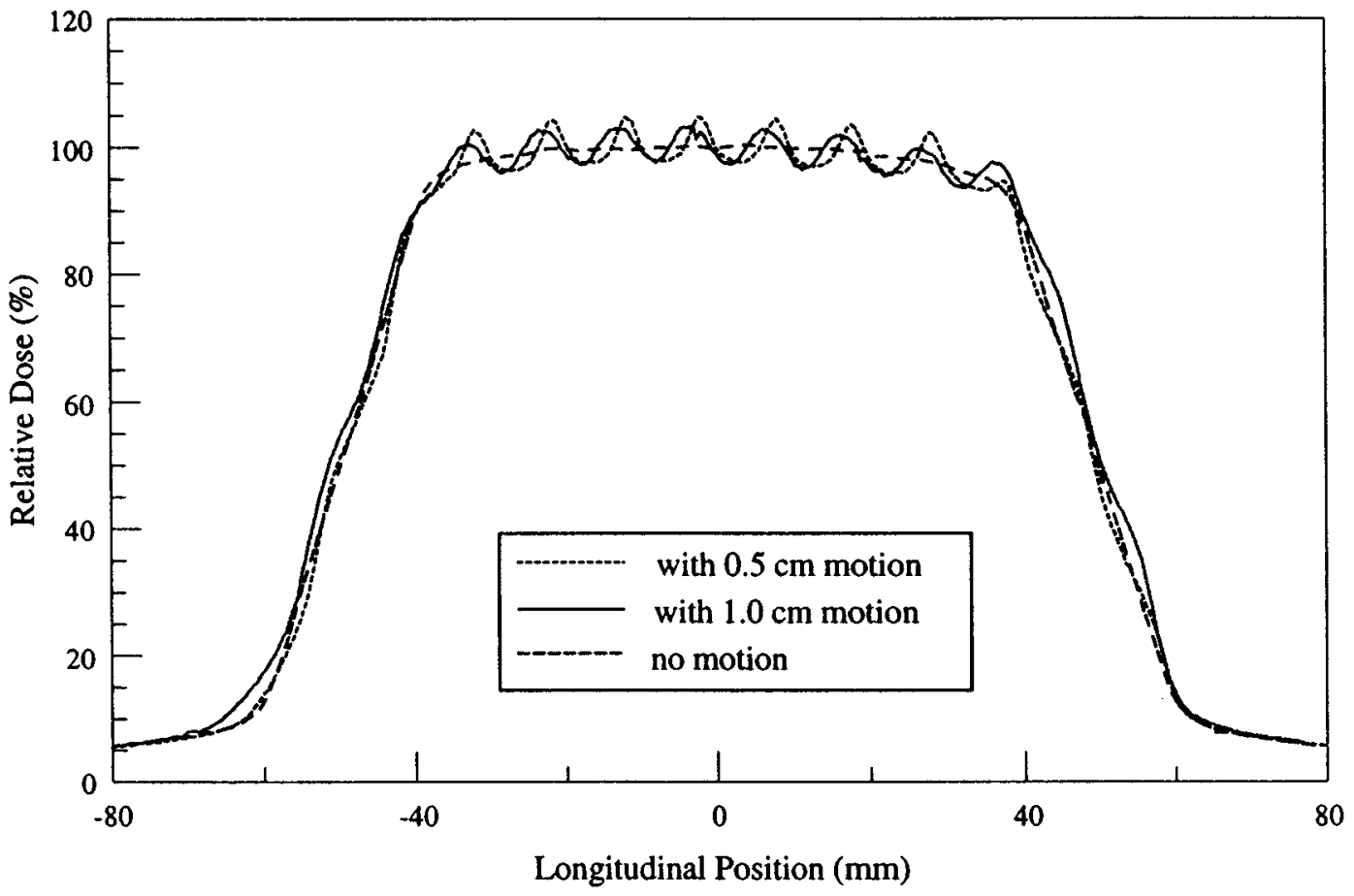

FIG. 9. The measured longitudinal dose profiles for helical beam delivery along the phantom axis of symmetry with simulated longitudinal target motion. The target motion frequency was eight cycles $/ \mathrm{min}$ with magnitudes of 0.5 or $1.0 \mathrm{~cm}$. The beam size was $8.0 \mathrm{~cm} \times 2.0 \mathrm{~cm}$. The helical beam was rotated at $4 \mathrm{rpm}$, and linearly translated at $2.0 \mathrm{~cm} /$ rotation. The running start/stop was not performed.

continuous helical beams at the center of the irradiated volume measuring $5.0 \mathrm{~cm} \times 8.0 \mathrm{~cm}$ and $8.0 \mathrm{~cm} \times 8.0 \mathrm{~cm}$ (the first volume dimension is in the transverse direction). The slit beam field sizes were $5.0 \mathrm{~cm} \times 2.0 \mathrm{~cm}$ and $8.0 \mathrm{~cm} \times 2.0 \mathrm{~cm}$ correspondingly.

Figure 9 displays measured longitudinal dose profiles at the phantom central axis with longitudinal oscillating tumor motion during the helical beam irradiation. The $2 \mathrm{~cm}$ wide helical slit beam moved at $4 \mathrm{rpm}$ simultaneously with 2.0 $\mathrm{cm} / \mathrm{rev}$ longitudinal translation speed. The frequency of the simulated target motion was 8 cycles/min, which was smaller than typical breathing rates. The magnitudes of the target oscillation were 0.5 or $1.0 \mathrm{~cm}$. Since only the relative motion between the beam and target is determinative, this would also be the result for any case with the same ratio of the target motion frequency and the helical beam rotation frequency while all other parameters remain unchanged. The relative off-axis dose profiles in the central region with motion were nearly identical, except there was a very small phase shift in the dose perturbations.

Figure 10 shows the measured longitudinal dose profiles for $2 \mathrm{~cm}$ width helical beams with a $1.0 \mathrm{~cm}$ magnitude breathing motion at different frequencies ranging from 5 to 20 breaths per minute. The profiles for higher breathing frequencies were very close to the profile without motion. The greatest perturbation was for the target speed that was closest to the helical beam longitudinal speed.

Figure 11 shows the measured longitudinal dose profiles with simulated target motion for helical beams of different slit widths. The simulated breathing motion was $1.0 \mathrm{~cm}$ in magnitude and 10 cycles/min in frequency. The slit widths were $0.5,1.0$, and $2.0 \mathrm{~cm}$. The longitudinal dose profile with 0.5 and $1.0 \mathrm{~cm}$ slit widths were very close to that of the non-breathing-motion helical beam. For the $2.0 \mathrm{~cm}$ slit width, the flatness was worse, but was still within $\pm 2 \%$ in most regions, excluding the dose fall-off regions. As the figure shows, the smaller the field width, the sharper the penumbra of the total irradiated volume. However, with the running start/stop technique implemented, there should be little or no penumbra width variation as a function of field width.

The experimental studies were also carried out for SST. The measured longitudinal dose profiles for different field widths, with or without motion, are shown in Figs. 12(a) and 12(b). For a $\pm 1 \mathrm{~mm}$ difference in the field width and the couch advancement (i.e., the index error), a $\pm 15 \%-20 \%$ hot or cold regions would be developed at the junction of the segments for a static target. The motion served to effectively blur the beam junction for each segment so that the field abutting position was less critical. The extension of the penumbra can be better observed for the $2.0 \mathrm{~cm}$ field length (Fig. 13), where motions with 10 and 20 cycles/min almost had the same effect.

Figure 14 shows the measured longitudinal dose profiles for helical beams with a simulation of the discretely delivered running start/stop technique implemented using static fields of different slit widths to sharpen the inferior-superior dose fall-off region. Between the running start and the running stop regions, the helical beam dose profile was continuous and obtained in the same fashion of that of Fig. 5. A 


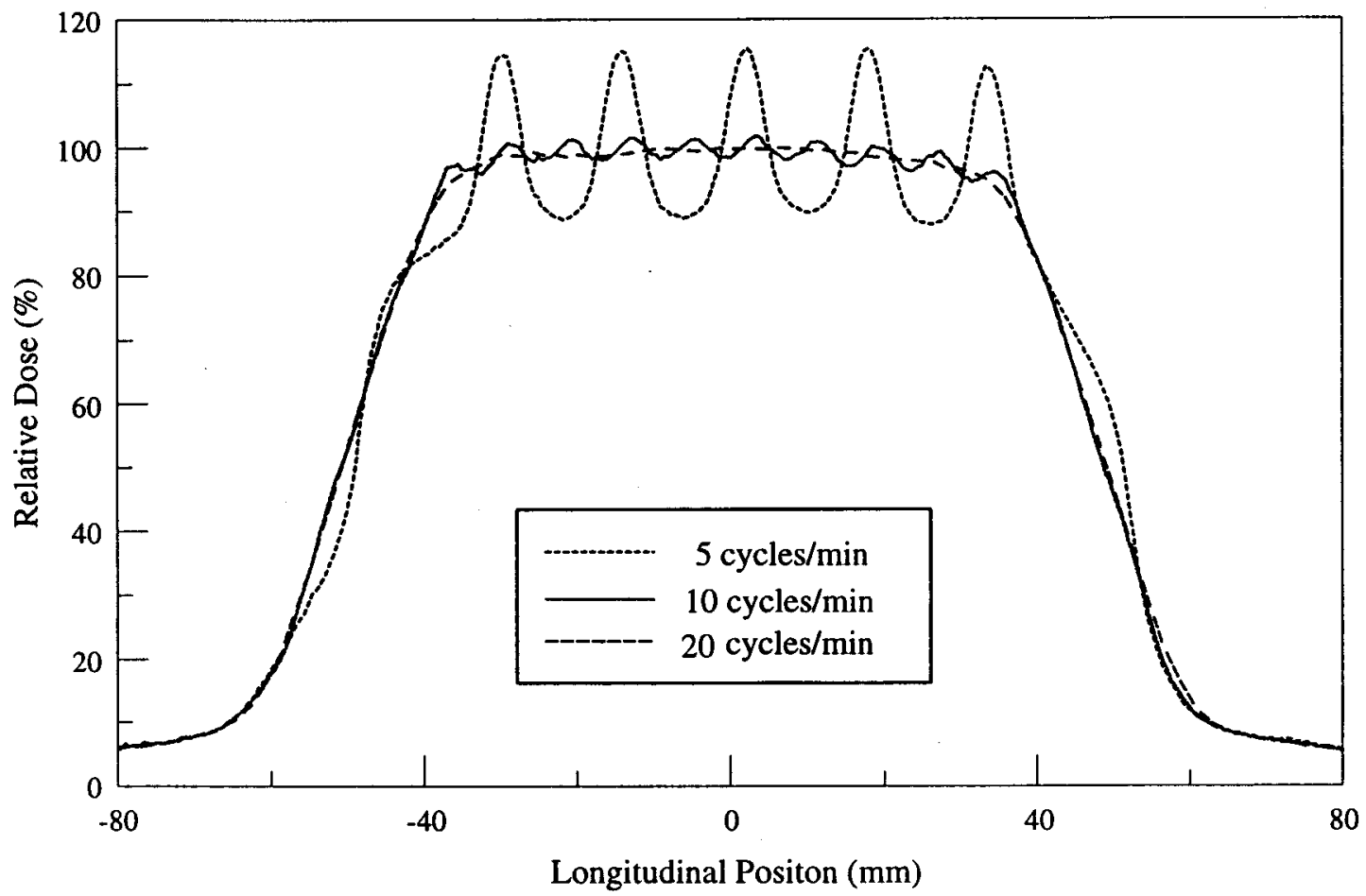

FIG. 10. The measured longitudinal dose profiles along the phantom symmetric axis for helical beams with simulated longitudinal target motion. The simulated target motion magnitude was $1.0 \mathrm{~cm}$. Motion frequencies were 5,10 , and 20 cycles/min, corresponding to the curves in order of decreasing deviations in the central region. The beam size was $8.0 \mathrm{~cm} \times 2.0 \mathrm{~cm}$. The beam was rotated at $4 \mathrm{rpm}$ and linearly translated at $2.0 \mathrm{~cm} / \mathrm{rotation}$. The running start/stop was not performed.

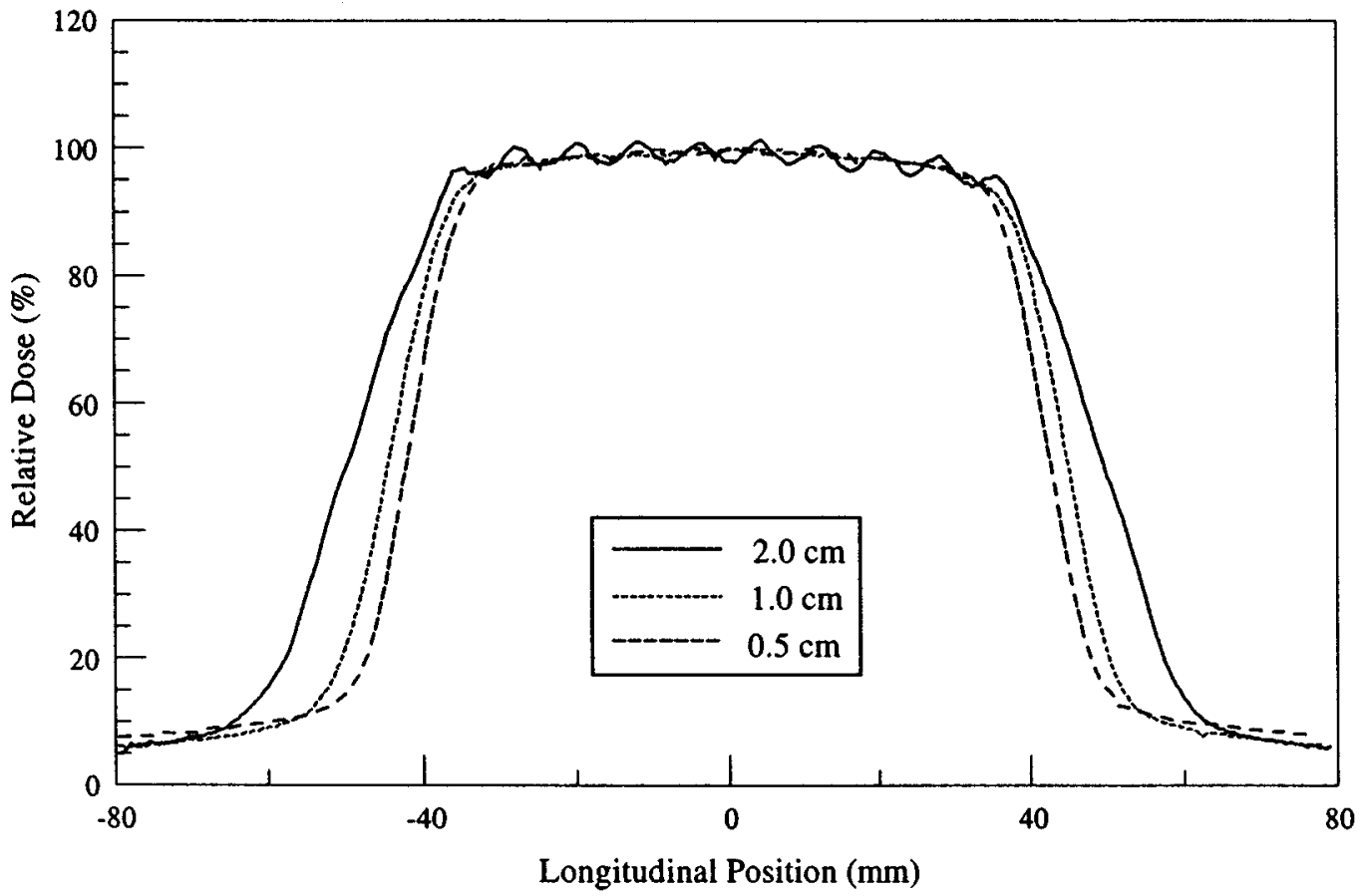

FIG. 11. The measured longitudinal dose profiles along the phantom symmetric axis for helical beams with simulated longitudinal target motion of ten cycles $/ \mathrm{min}$ and $1 \mathrm{~cm}$ magnitude for different field widths: $2.0,1.0$, and $0.5 \mathrm{~cm}$. The field length is $8 \mathrm{~cm}$. The running start/stop was not performed. 
(a)

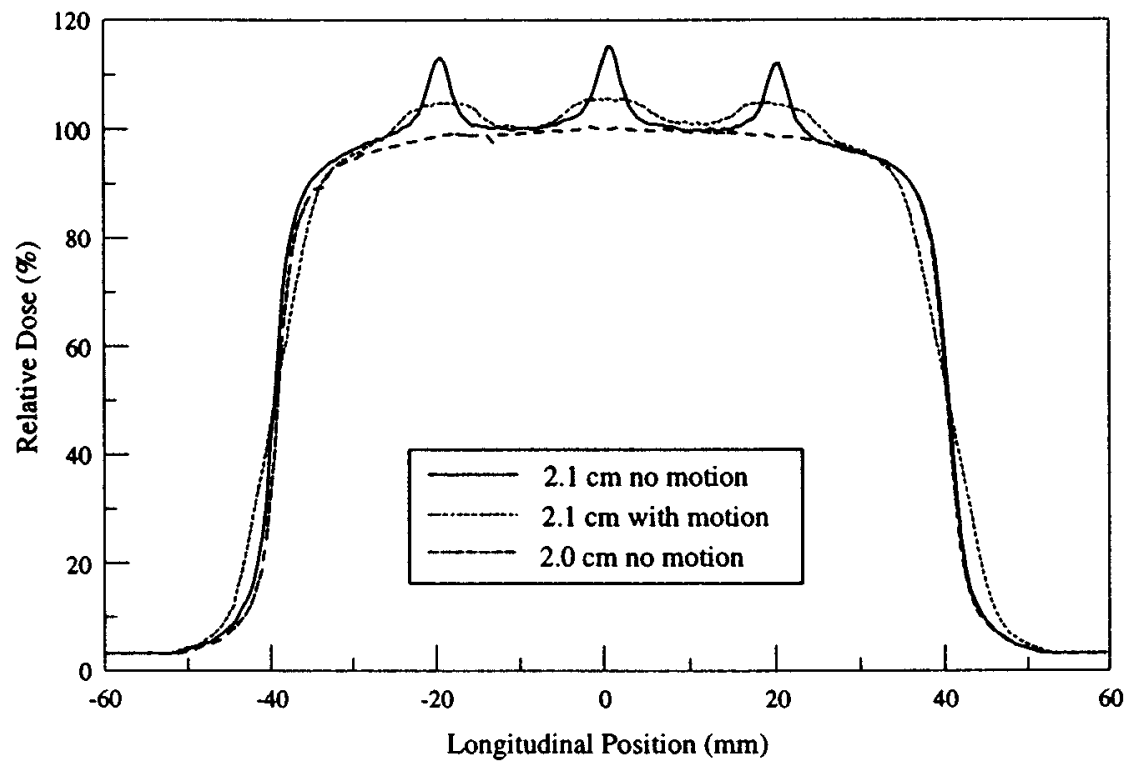

(b)

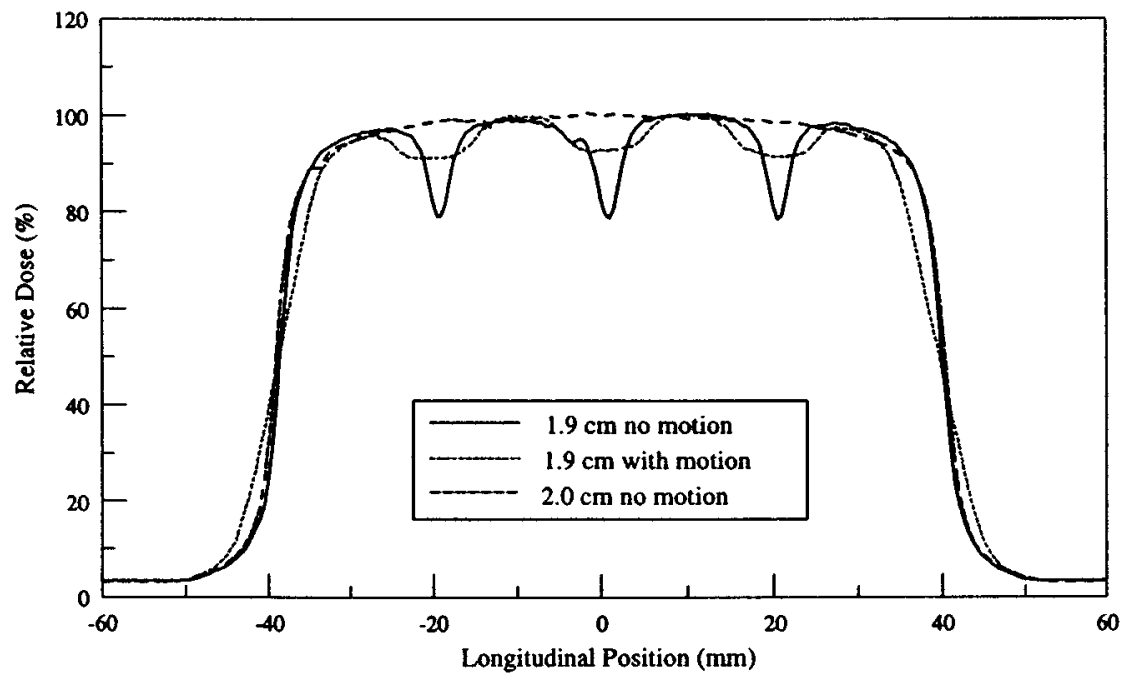

FIG. 12. The measured longitudinal dose profiles along the phantom symmetric axis for sequential beams with and without simulated longitudinal target motion of 10 cycles $/ \mathrm{min}$ and $1 \mathrm{~cm}$ magnitude for fields with different beam width but the same longitudinal translational distance of $2.0 \mathrm{~cm}$. The profile for a $2.0 \mathrm{~cm}$ slit beam without motion is shown as a reference. (a) is for a $2.1 \mathrm{~cm}$ beam width. (b) is for a $1.9 \mathrm{~cm}$ beam width.

profile for a helical beam without using this technique is shown in this figure for comparison.

\section{DISCUSSION}

The dose profile for a complete rotation of helical beam delivery (Fig. 5) represents a basic element of helical beam dosimetry. For the ease of discussion, we can imagine that this beam profile is produced by a conventional beam with a beam modifier so that the profile has a triangular shape. Then the helical tomotherapy delivery could be viewed as a SST delivery with such a triangular-shaped beam. The linearity and slow dose fall-off determined that the field matching between adjacent SST segments of this hypothetical beam would not be position sensitive. It almost guaranteed a uni- form longitudinal dose distribution as long as the matching point is within a few millimeters of the $50 \%$ dose level and the matching point is consistent through the whole region. In other words, the systematic errors in the collimator setting or couch motion would not perturb the relative dose uniformity delivered by such a beam. The absolute dose level may change approximately directly proportional to the field width and inversely proportional to couch velocity. However, an accurate collimator and couch velocity are much easier to assure than junction matching. The dose uniformity as exhibited by longitudinal (Fig. 6) and transverse dose profiles (Fig. 7) for helical beam delivery were adequate in the intended treatment area. With intensity modulation, the transverse dose fall-off would be made sharper, where it is required to 


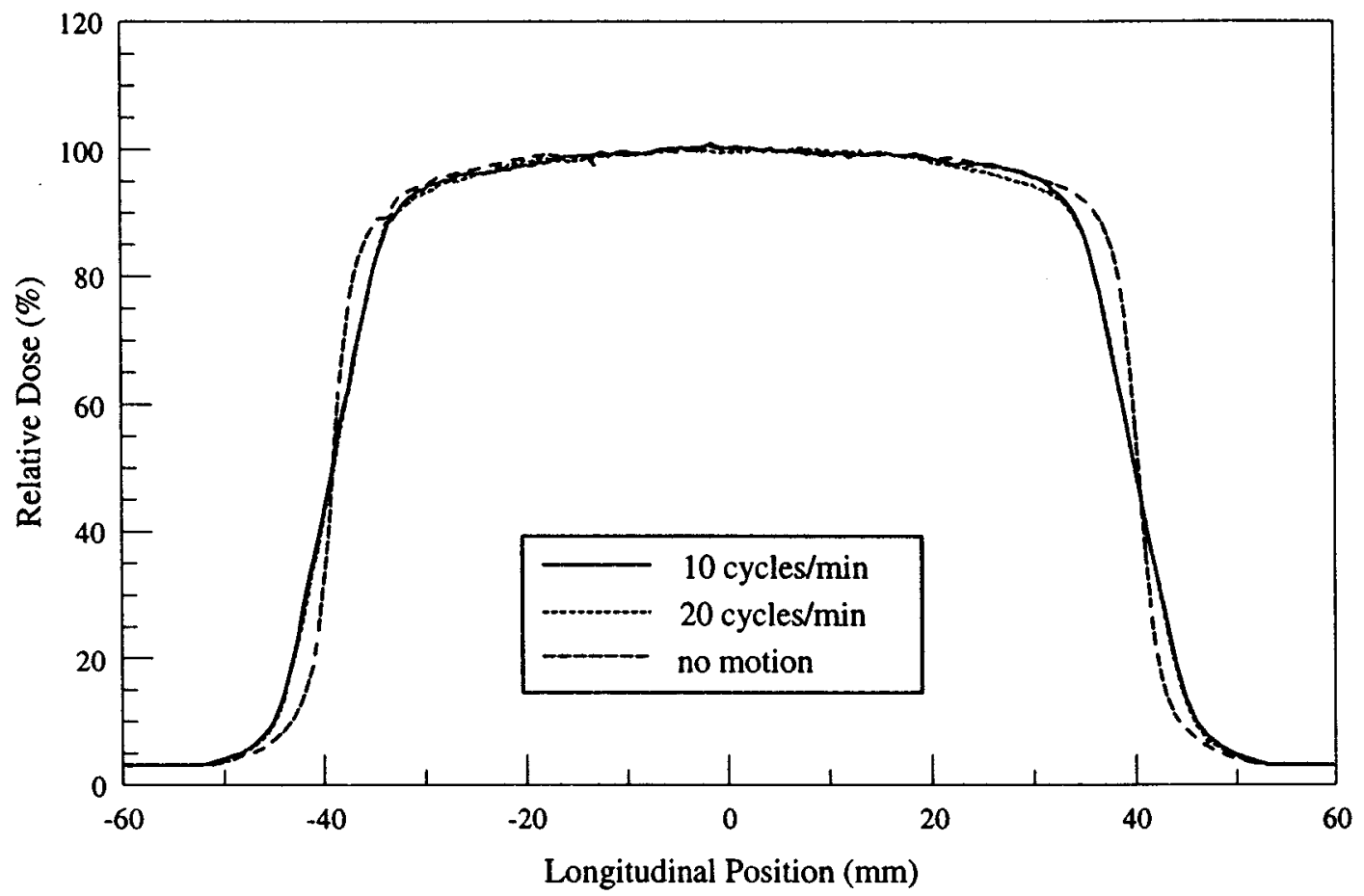

FIG. 13. Longitudinal dose profiles along the phantom axis for sequential beams with $1.0 \mathrm{~cm}$ magnitude and 10,20 cycles/min target motions. The field size is $8.0 \mathrm{~cm} \times 2.0 \mathrm{~cm}$. The curve with the sharper fall-off is the profile without motion.

spare critical normal tissue, and less steep where there is no neighboring critical structures. This is mainly because less primary beam intensity would directly pass through critical structure and more would pass through noncritical regions.
The SST can deliver a uniform dose distribution for a perfectly matched sequential rotational beam. The traditional field boundary matching as used in the SST technique requires high precision due to its sharp fall-off (Fig. 4). Since

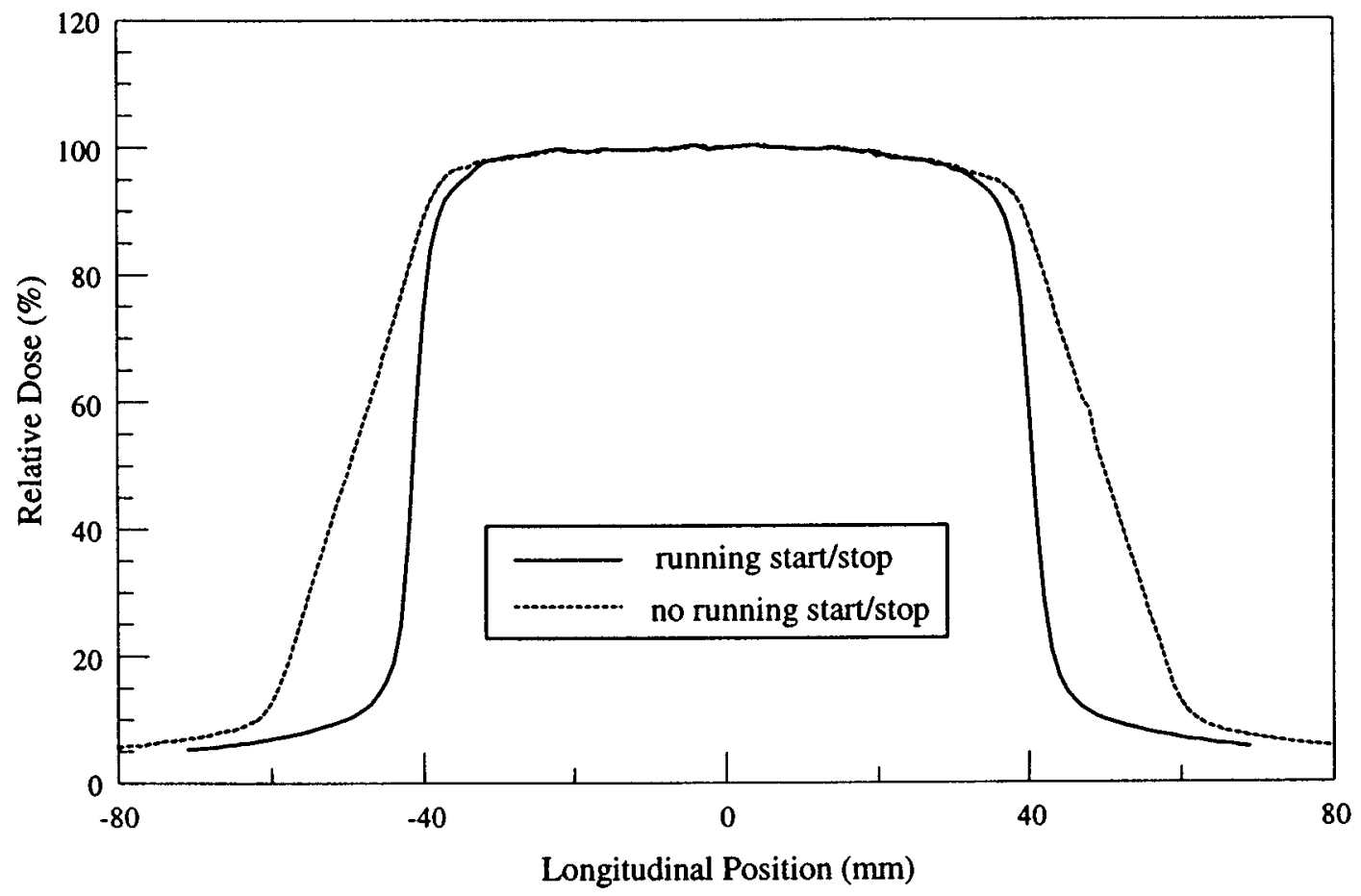

FIG. 14. Longitudinal dose profiles along the phantom axis for a helical beam with the running start/stop technique. The field size was $8.0 \mathrm{~cm} \times 2.0 \mathrm{~cm}$, except during the start/stop phase, where ten static beams of various slit widths per rotation used as an approximation for the continuous running start/stop technique. The curve with the larger penumbra was the corresponding dose profile without the running start/strop technique. 
the helical beam is continuously advancing, no interruption is needed before the whole irradiation could be delivered. This eliminates the possible human positioning errors and couch index errors involved in the SST technique, in which a treatment would be frequently interrupted in order to adjust patient position after each segment. Should such an error occur in SST, the dose uniformity would be severely perturbed, as could be seen in Fig. 12 (curves with no motion), where a $\pm 0.1 \mathrm{~cm}$ error in the actual $\mathrm{x}$-ray field length and the translation distance can result in up to a $20 \%$ deviation from the desirable dose. This was due to the sharpness of the dose fall-off of the slit beam. However, the $1 \mathrm{~mm}$ difference in field width did not affect relative dose uniformity in helical beam delivery. The absolute dose could be maintained at the same level by adjusting the monitor units accordingly.

The longitudinal breath motion did nothing but extend slightly the dose fall-off at the superior-inferior boundaries for the perfect matched SST (Fig. 13). However, if the match is off by $\pm 0.1 \mathrm{~cm}$, only a $\pm 7 \%$ dose variation would result (Fig. 12). It was much better than variations without the target motion for the SST technique. This indicates that small longitudinal blurring mechanically produced by the collimator or couch could reduce the magnitude of possible junctioning artifacts.

Our motion studies have also shown, for motions typical of excursions due to breathing, that the dose fluctuation was less than $\pm 5 \%$ for a single treatment for helical beam delivery for a $2 \mathrm{~cm}$ slit width helical beam rotated at $4 \mathrm{rpm}$. There was a larger variation in dose profile for frequencies that are closest to the rotation frequency and higher amplitudes. The closest frequency in our experiment was five cycles/min. Breathing frequency at or less than eight times per minute would be atypical. With normal breathing frequency greater than eight cycles/min, the helical beam rotating at $4 \mathrm{rpm}$ is acceptable. It is not hard to see that the dose perturbation depends on the relative speed between the target motion and longitudinal translational speed of the slit beam. The hottest/ coldest spot would be produced when they have the same speed at the lowest frequency and highest magnitude of breath motion. This would cause the longest irradiation time at a spot when they move in the same direction and shortest irradiation time at another spot when they travel in the opposite directions. The dose perturbations reduced rather rapidly with an increasing difference in the longitudinal speeds of the target and slit beam. For slower breathing frequencies, the helical beam rotation frequency and/or slit beam width may need to be adjusted appropriately to reduce the dose perturbations. The location of the hot or cold spot depends on the relative phase difference between helical motion and target motion. Assuming that the phase difference for each treatment is randomly distributed, the total dose distribution for a typical 20-fraction treatment will be much smoother than the single fraction distribution. For a fixed breathing frequency, a slight difference in motion magnitude did not affect the dose variation much. For a fixed beam rotational speed and a fixed target oscillation magnitude, the higher the oscillation frequency, the smoother the dose profile (see Fig. 10). This suggests that, for a certain magnitude and fre- quency of motion, there will be an upper limit for the rotational speed of the beam to achieve a satisfactory uniform dose in the target. This limit will also be affected by the field width. This is because the field width and the rotational speed of the beam determine the linear translational speed of the phantom or patient. For the helical beam, rapid motions such as the beating heart would not affect the longitudinal dose uniformity, since the frequency of the motion would be much higher than the rotation frequency of the helical slit beam.

As shown in Fig. 14, a simple discrete running start/stop technique as implemented here could reduce the $20 \%-80 \%$ fall-off from 15 to $5 \mathrm{~mm}$. The latter is better or the same as a similar dose fall-off region for a typical $6 \mathrm{MV}$ field in conventional radiotherapy. Though the discrete running start/ stop will not be used in real clinical treatment, it provides an experimental confirmation. A dynamic running start/stop implementation is expected to achieve a similar effect, if not better.

\section{CONCLUSIONS}

Helical beam delivery, as proposed by Mackie et al., ${ }^{15}$ could deliver uniform dose distributions in the target volume, both longitudinally and transversely. The running start/stop technique could sharpen the overall longitudinal dose fall-off regions dramatically, making it comparable to that of conventional large field rotational therapy. Periodic linear motions with amplitudes and frequencies of typical breathing motions caused minor dose variations for the helical beam with a $2 \mathrm{~cm}$ slit beam and a $4 \mathrm{rpm}$ rotational speed. If we apply these results to irradiation of the thorax, they suggest that normal or rapid breathing will result in better dose uniformity than slow breathing. For those patients who are unable to breathe in this fashion, a larger field width and/or a slower gantry rotation may reduce the artifacts.

These results can broadly be generalized to all forms of radiotherapy with intensity modulation. Motion will cause artifacts both in the central and fall-off regions. The extent of the effects will depend on the degree of modulation and the character of the motion.

\section{ACKNOWLEDGMENTS}

This work was partially supported by NCI Grant No. CA48902 and NRSA Grant No. T32CA09206.

\footnotetext{
${ }^{1}$ A. Brahme, J-E. Roos, and I. Lax, "Solution of an integral equation encountered in rotation therapy," Phys. Med. Biol. 27, 1221-1229 (1982).

${ }^{2}$ A. M. Cormack, "A problem in rotation therapy with X-rays," Int. J. Radiat. Oncol. Biol. Phys. 13, 623-639 (1987).

${ }^{3} \mathrm{~A}$. Brahme, "Optimization of stationary and moving beam radiation therapy techniques," Radiother. Oncol. 12, 129-140 (1988).

${ }^{4}$ N. H. Barth, "An inverse problem in rotation radiotherapy,' Int. J. Radiat. Oncol. Biol. Phys. 18, 425-431 (1990).

${ }^{5} \mathrm{H}$. Kooy and N. H. Bath, "The verification of an inverse problem in radiation therapy,' Int. J. Radiat. Oncol. Biol. Phys. 18, 433-439 (1990). ${ }^{6} \mathrm{~T}$. Bortfeld, J. Bürkelbach, R. Boesecke, and W. Schlegel, "Methods of image reconstruction from projections applied to conformation radiotherapy,"' Phys. Med. Biol. 35, 1423-1434 (1990).
} 
${ }^{7} \mathrm{~S}$. Webb, "Optimization of conformal radiotherapy dose distribution by simulated annealing," Phys. Med. Biol. 34, 1349-1370 (1989).

${ }^{8} \mathrm{~S}$. Webb, "Optimization by simulated annealing of three-dimensional conformal treatment planning for radiation fields defined by a multileaf collimator," Phys. Med. Biol. 36, 1201-1226 (1991).

${ }^{9} \mathrm{~S}$. Webb, "Optimization of conformal radiotherapy dose distributions by simulated annealing: 2. Inclusion of scatter in the 2D technique," Phys. Med. Biol. 36, 1227-1237 (1991).

${ }^{10} \mathrm{~S}$. Webb, "Optimization by simulated annealing of three-dimensional conformal treatment planning for radiation fields defined by a multileaf collimator: II. Inclusion of two-dimensional modulation of the x-ray intensity,"' Phys. Med. Biol. 37, 1689-1704 (1992).

${ }^{11}$ B. K. Lind, "Radiation therapy planning and optimization studied as inverse problems,"' Ph.D. thesis, Stockholm University, Stockholm, 1991.

${ }^{12}$ T. W. Holmes, T. R. Mackie, D. J. Simpson, and P. Reckwerdt, "A unified approach to the optimization of brachytherapy and external beam dosimetry,' Int. J. Oncol. Biol. Phys. 20, 859-873 (1991).

${ }^{13}$ T. Holmes and T. R. Mackie, "A filtered backprojection dose calculation method useful for inverse treatment planning," Med. Phys. 21, 303-313 (1991).

${ }^{14} \mathrm{~T}$. Holmes and T. R. Mackie, "A comparison of three inverse treatment planning algorithms," Phys. Med. Biol. 39, 91-106 (1994).

${ }^{15}$ T. R. Mackie, T. Holmes, S. Swerdloff, P. Reckwerdt, J. O. Deasy, J. Yang, B. Paliwal, and T. Kinsella, "Tomotherapy: A new concept for the delivery of dynamic conformal radiotherapy," Med. Phys. 20, 17091719 (1993).

${ }^{16}$ M. P. Carol, "Beam modulation conformal radiotherapy," in 3D Radiation Treatment Planning and Conformal Therapy, edited by J. A. Purdy, and B. Emami (Medical Physics Publishing, Madison, WI, 1995).

${ }^{17}$ A. L. Boyer and T. Schultheiss, "Effects of dosimetric and clinical uncertainty on complication-free local tumor control," Radiother. Oncol. 11, 65-71 (1988).
${ }^{18}$ C. Ross, D. H. Hussey, E. C. Pennington, W. Stanford, and F. Doombos, "Analysis of movement of intrathoracic neoplasms sing ultrafast computerized tomography," Int. J. Radiat. Oncol. Biol. Phys. 18, 671-677 (1990).

${ }^{19}$ Y. Onogi, Y. Aoki, K. Nakagawa, A. Akanuma, and Y. Sasaki, “A new method for target evaluation with organ movement," Proceedings of the XIth International Conference On The Use of Computers in Radiation Therapy (Christie Hospital NHS Trust, Manchester, UK, 1994).

${ }^{20}$ A. L. Boyer, T. R. Bortfeld, D. L. Kahler, and T. J. Waldron, "Multileaf collimation for 3-D conformal radiotherapy," in Ref. 16.

${ }^{21}$ D. J. Convery and M. E. Rosenbloom, "The generation of intensitymodulated fields for conformal radiotherapy by dynamic collimation," Phys. Med. Biol. 37, 1359-1374 (1992).

${ }^{22}$ B. A. Fraass, D. L. McShan, M. K. Kessler, G. M. Matrone, and T. Weaver "Computer-controlled conformal radiation therapy," in Ref. 16.

${ }^{23}$ A. S. Zacarias, R. G. Lane, and I. I. Rosen, "Assessment of a linear accelerator for segmented conformal radiation therapy," Med. Phys. 20, 193-198 (1993).

${ }^{24}$ Parker Hannifin Corporation, "Daedal manual \& motorized positioning systems," Harrison City, PA.

${ }^{25}$ T. W. Holmes, "A model for the physical optimization of external beam radiotherapy," Ph.D. thesis, University of Wisconsin (Medical Physics Publishing, Madison, WI, 1993).

${ }^{26}$ T. J. Davy, P. K. Johnson, R. Redford, and J. R. Williams, "Conformation therapy using the tracking cobalt unit," Br. J. Radiol. 48, 122-130 (1975).

${ }^{27}$ T. J. Davy, "Physical aspects of conformation therapy using computercontrolled tracking units," in Progress in Medical Radiation Physics, edited by C. G. Orton (Plenum, New York, 1985), Vol. 2.

${ }^{28} \mathrm{~W}$. A. Jennings, "The tracking cobalt project: From moving-beam therapy to three-dimensional programmed irradiation," in Ref. 27. 